\title{
DC-link capacitor sizing method for a wireless power transfer circuit to be used in drone opportunity charging
}

\author{
Andrea Carloni, Federico Baronti, Roberto Di Rienzo, \\ Roberto Roncella, Roberto Saletti \\ Dipartimento di Ingegneria dell'Informazione, University of Pisa, \\ via Girolamo Caruso,16, 56122 Pisa, Italy \\ andrea.carloni@ing.unipi.it
}

\begin{abstract}
Resonant-coupled inductive Wireless Power Transfer systems are very appealing as opportunity charging systems for drone applications. Drones are compact systems in which weight and size are critical constraints, so the on-board electronics and the battery must be as small and light as possible. The paper presents the LTSpice simulation analysis of a circuit on the WPT secondary side that uses the intrinsic inductance of the Li-poly battery and only an external capacitor as filter of the full-wave bridge rectifier that typically constitutes the DC-link. The analysis shows the trade-off between the power delivered to the battery and the capacitor size. It results that it can be found a capacitor value that maximizes the power transfer to the battery at the expense of a non-optimal transfer efficiency and increased ripple in the battery current. That value sets the $L C$-filter resonant frequency close to the double of the excitation frequency of the WPT system.
\end{abstract}

\section{Introduction}

The good mid-range power transfer capabilities achievable with resonant-coupled inductive Wireless Power Transfer (WPT) systems have led to many battery opportunity charging implementations for application to flying devices such as drones [1]. A basic WPT system consists of two magnetically coupled resonant circuits called primary and secondary circuits. Usually, the primary circuit design is straightforward, as it is not limited by weight and volume constraints. It is powered by an external energy source and employs a D-class amplifier [2]. Instead, the secondary circuit design is critical as it is located on board the drone, where weight is a major issue. It is mainly made by a bridge rectifier that supplies the battery to be recharged [3]. Depending on the application type and the quality of the WPT system, a DC-DC converter and/or filters are present in the secondary side to rectify the transmitted AC power and to control the charge of the battery. The authors reach and track the maximum charging efficiency using a DC-DC converter with variable duty-cycle in [4]. However, this architecture might be not affordable for drones where the overall dimensions and weight are important constraints. Therefore, a simpler solution based on an $L C$-filter may be preferable. The DC-link $L C$-filter architecture used in [5] is safely sized by choosing the capacitor value $C$ large enough to obtain a constant output DC voltage on it. However, this assumption may lead to overestimate the capacitor value and to add 
useless size and load to the drone. The capacitor and inductor sizes are related to the maximum current and voltage values that they can withstand, so these factors can be critical when the power requirement of the opportunity charger becomes large. Moreover, as WPT systems usually work with resonant frequencies from tens of $\mathrm{kHz}$ to $\mathrm{MHz}$ [3], the available capacity values of commercial capacitors are much less than those that work at lower frequencies. The aim of this paper is to investigate how the sizing of the filtering capacitor influences the power transfer and the efficiency of a generic WPT, by means of LTSpice time-domain simulations. In addition, as a generic Li-ion battery shows an intrinsic inductive component in the WPT frequency range as shown in [6], our idea is to eliminate the external passive inductor and to use the battery itself as the inductive component of the $L C$-filter to achieve a further size and load saving. The final goal of the paper is to find the best trade-off between the size of the $L C$-filter capacitor and the power delivered to the battery.

\section{Methodology}

Fig. 1 shows the equivalent circuits for the series-series (SS) WPT architecture investigated in this paper. The secondary circuit consists of the diode rectifier bridge and only the passive component $C_{0}$, whereas $R_{B}$ and $L_{B}$ are the intrinsic parameter of the Randal model [6] of a generic Li-ion battery valid at the frequencies of interest. Let us determine the frequency response of the $L C$-filter consisting of $C_{0}$ and the parasitic inductance of the battery.

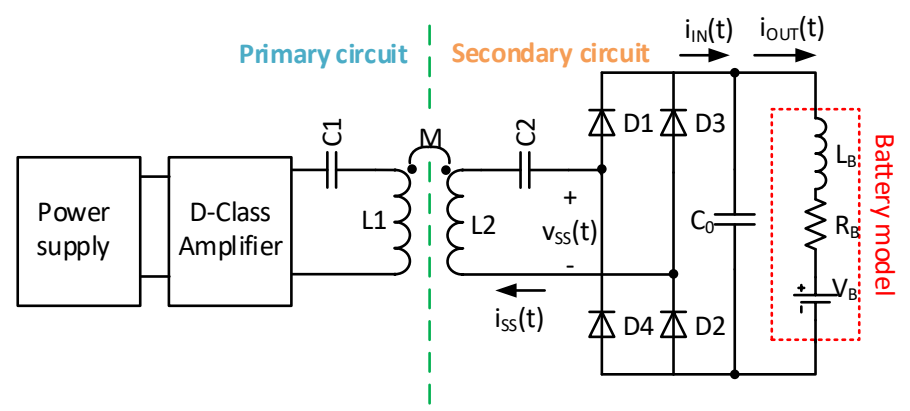

Fig. 1. Equivalent circuit of the SS WPT architecture proposed in this paper.

\subsection{LC -filter Frequency Response}

As described in [7], the SS architecture fixes the current in the secondary circuit. Therefore, the frequency response of the $L C$-filter showed in Fig. 1 can be evaluated by considering the current $i_{I N}(t)$ coming from the rectifier bridge as input, and the current $i_{\text {OUT }}(t)$ that flows in the Li-ion battery as output. The circuit behaves like a second order low-pass filter, the Laplace domain response of which is well known and shown in (1), together with the discriminant $\Delta$ of the polynomial [8]. The value of $\Delta$ determines the position of the poles of the filter. The value of $C_{0}$ that makes $\Delta=0$, i.e. 
$C^{*}$ reported in (2), sets the limit between real and complex conjugate poles. If $C_{0}$ is lower than $C^{*}$, the filter has complex conjugates poles.

$$
\begin{gathered}
\frac{I_{\text {OUT }}(s)}{I_{I N}(s)}=\frac{1}{s^{2} L_{B} C_{0}+s R_{B} C_{0}+1} \quad \Delta=C_{0}\left[R_{B}^{2} C_{0}-4 L_{B}\right] \\
C^{*}=\frac{4 L_{B}}{R_{B}{ }^{2}}
\end{gathered}
$$

By defining, $f_{0}$ as the resonant frequency and $\xi$ as the damping factor of the filter as expressed in (3),

$$
f_{0}=\frac{1}{2 \pi \sqrt{L_{B} C_{0}}}, \quad \xi=\frac{R_{B}}{2} \sqrt{\frac{C_{0}}{L_{B}}},
$$

equation (1) can finally be written as in (4)

$$
\frac{I_{O U T}(s)}{I_{I N}(s)}=\frac{1}{\frac{s^{2}}{4 \pi^{2} f_{0}^{2}}+\frac{\xi}{\pi f_{0}} s+1} .
$$

When the damping factor approaches to 0 , the time-domain response will show an increased oscillating behaviour.

\subsection{Time-Domain Simulation Analysis}

The time-domain response of the secondary circuit in Fig. 1 was evaluated as a function of the circuit parameters by means of the LTSpice electrical simulator. Since the SS WPT architecture behaves like a current generator [7], we represent the circuit up to the bridge rectifier as a sinusoidal current generator $i_{s s}(t)$, with $25 \mathrm{~A}$ amplitude and $150 \mathrm{kHz}$ frequency. These values resemble those commonly used in medium power applications such as drones [2],[9]. The diodes are modelled as ideal switches with a voltage drop $V_{\gamma}$ of $640 \mathrm{mV}$. The battery voltage is fixed at $22 \mathrm{~V}$, whereas $L_{B}$ and $R_{B}$ were extracted from a real Li-ion battery as it will described in the following subsection. The .step directive in LTSpice allows us to perform a parametric simulation, where the value of $C_{0}$ is logarithmically swept between $50 \mathrm{nF}$ and $500 \mu \mathrm{F}$. Finally, the total power $P_{B}$ transferred to the battery and the input-output efficiency $\eta$ are evaluated. Let us note that $P_{B}$ is the active power transferred to the battery, being defined as the average of the product between the battery electromotive force $V_{B}$ and the battery current $i_{O U T}(t)$ in Fig. 1. Moreover, $\eta$ is the ratio between $P_{B}$ and the power at the bridge rectifier input.

\subsection{Battery Parameter Experimental Extraction}

The intrinsic resistance and inductance of a real Li-ion battery specific for drone applications were measured by performing the Electrochemical Impedance Spectroscopy (EIS) of a TA-15C-16000-6S1P-EC5 battery. It consists of 6 cells in series, with $22.2 \mathrm{~V}$ nominal voltage and 16 Ah capacity. The spectroscopy test was 
performed by means of a Gamry Reference 3000 [10] set in galvanostatic mode. The instrument sets a $0.1 \mathrm{AC}$-current on the single cell and measures the cell voltage between $1 \mathrm{~Hz}$ and $500 \mathrm{kHz}$ in ten points per decade. The extracted Bode diagrams of the impedance were fitted by the Gamry Echem Analyst software [11] by which the intrinsic resistance and inductance of the six cells of the battery were derived.

\section{Results and Discussion}

The resistance values derived as above show an average value of $29.957 \mathrm{~m} \Omega$ with a standard deviation of $76.085 \mu \Omega$, whereas the average inductance is $217.3 \mathrm{nH}$, with $10.721 \mathrm{nH}$ standard deviation. Thus, the total battery resistance $R_{B}$ and inductance $L_{B}$ are $179.4 \mathrm{~m} \Omega$ and $1.304 \mu \mathrm{H}$, respectively. These values were used by the simulator to perform the analysis described in subsection 2.2. Furthermore, the value $C^{*}$ defined in (2) is $160.5 \mu \mathrm{F}$. The power transferred to the battery $P_{B}$ and the efficiency $\eta$ obtained by the circuit simulations when the capacitance $C_{0}$ is the parameter are shown in Fig. 2.

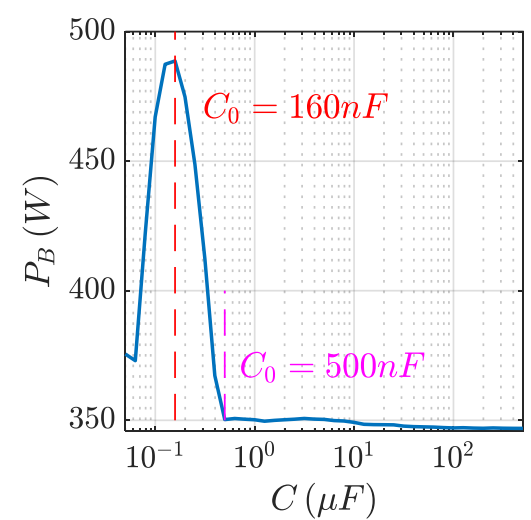

a)

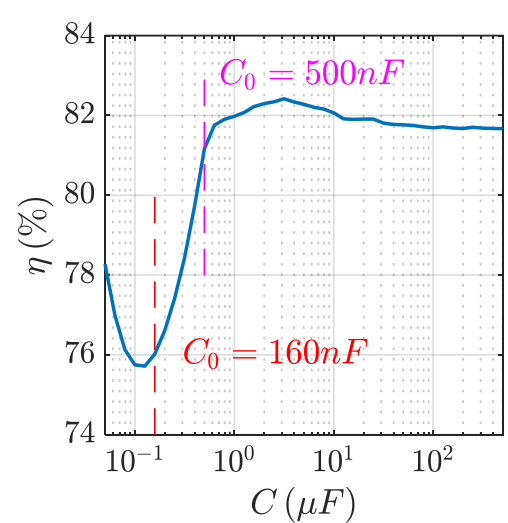

b)

Fig. 2 a) Total power transferred to the battery; b) Power efficiency.

It results that the power delivered to the battery and the circuit efficiency significantly depend on the value of $C_{0}$ and thus on the resonant frequency $f_{0}$ of the $L C$-filter. Considering the power graph in Fig. $2 \mathrm{a}$, it is possible to determine three intervals, where the circuit has three different behaviors. Fig. 3 shows the current and voltage waveforms at the rectifier input for three $C_{0}$ values, (a), (b) and (c), respectively, each one representing a particular behavior. Fig. 3 also shows the current waveforms at the rectifier input (red), rectifier output (blue) and in the battery (yellow), for the same three $C_{0}$ values, in (d), (e) and (f), respectively.

For capacity values higher than $500 \mathrm{nF}$ and thus for resonant frequencies lower than $197 \mathrm{kHz}$, the power is almost constant at $350 \mathrm{~W}$. The bridge rectifier works in continuous mode in this capacity interval. Its input voltage $v_{S S}(t)$ resembles a square wave as shown in Fig. 3a; the diodes D3, D4 and D1, D2 work as rectifying couples, and the filter provides good low-pass effect on the battery current [see Fig. 3(d)]. 


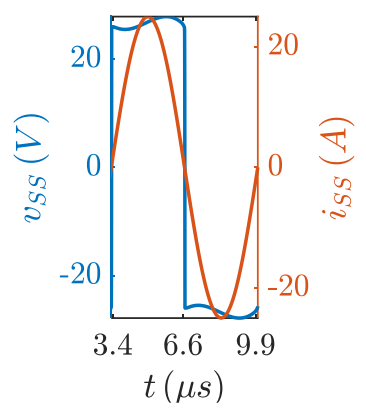

(a)

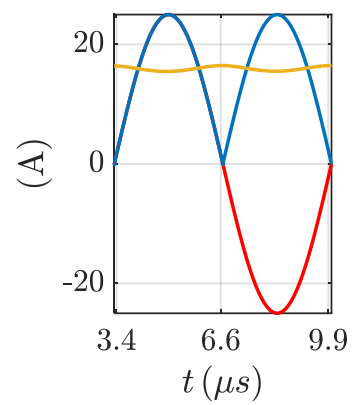

(d)

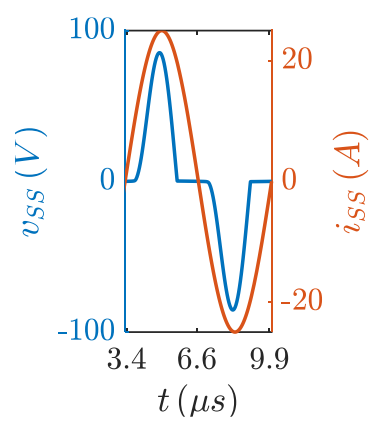

(b)

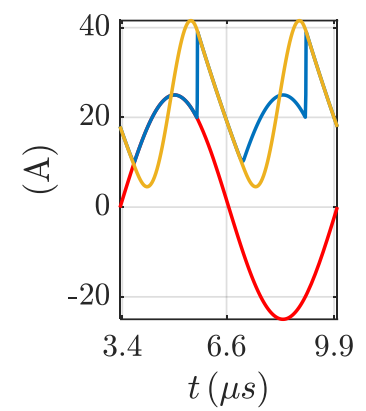

(e)

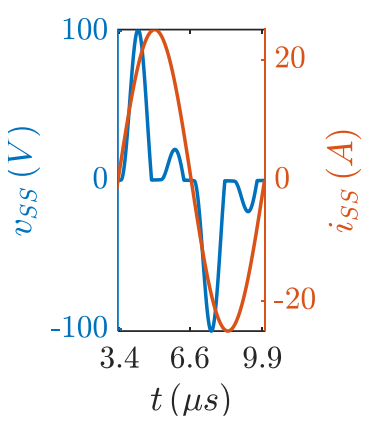

(c)

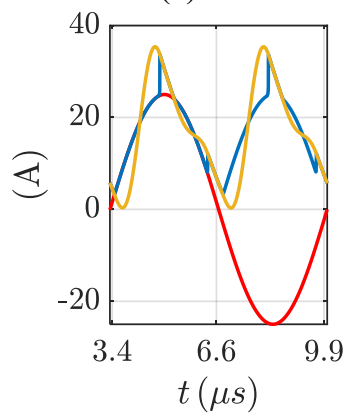

(f)

Fig. 3. Voltage $v_{S S}(t)$ and current $i_{S S}(t)$ waveforms at the input of the bridge rectifier, with $C_{0}=5 \mu \mathrm{F}(\mathrm{a}), C_{0}=126 \mathrm{nF}(\mathrm{b})$ and $C_{0}=50 \mathrm{nF}$ (c). Bridge rectifier input current $i_{s s}(t)$ (red line), bridge rectifier output current $i_{I N}(t)$ (blue line) and battery current $i_{\text {OUT }}(t)$ (yellow line) for $C_{0}=5 \mu \mathrm{F}(\mathrm{d}), C_{0}=126 \mathrm{nF}(\mathrm{e})$ and $C_{0}=50 \mathrm{nF}(\mathrm{f})$.

Instead, the power delivered to the battery grows for $C_{0}$ values between $500 \mathrm{nF}$ and about $160 \mathrm{nF}$, i.e. for $f_{0}$ between $197 \mathrm{kHz}$ and $349 \mathrm{kHz}$, as it can be seen in Fig. $2 \mathrm{a}$. We note that the bridge rectifier now works in discontinuous mode. There are time intervals in each semi-period of Fig. $3 \mathrm{~b}$, where $v_{S S}(t)$ is fixed at $-2 V_{\gamma}$, because all the diodes of the bridge simultaneously conduct. However, a beneficial effect is that $v_{S S}(t)$ is more like a sine wave than before, and the active power delivered to the load is higher with respect to the previous case. As $C_{0}$ approaches $160 \mathrm{nF}$, the phase angle between the fundamental components of $v_{S S}(t)$ and $i_{S S}(t)$ reduces itself, and the power delivered increases. This is a very appealing behavior, particularly for opportunity charging, where the goal is to deliver the maximum power possible. The drawback is found in the reduced filtering action, as the battery current becomes more oscillating [see Fig. 3(e)]. However, this fact does not affect the battery ageing as demonstrated in [12].

Then, the power starts to decrease when the capacity becomes lower than $160 \mathrm{nF}$. Finally, for capacity lower than $50 \mathrm{nF}$ and resonant frequency above $624 \mathrm{kHz}$, the waveforms in Fig. 3c and the battery current in Fig. 3f exhibit consistent overshoots and undershoots. This is a region to avoid, as the damping coefficient drops below than 0.02 . The efficiency profile in Fig. $2 \mathrm{~b}$ can also be divided in three sections with capacity intervals similar to the previous ones. The efficiency is quite constant at $80 \%$ for $C_{0}$ higher than $500 \mathrm{nF}$. Here, the $i_{\text {OUT }}(t)$ ripple is very low and produces a negligible loss on the resistance of the battery. For capacity lower than $500 \mathrm{nF}$, the efficiency starts to 
decrease, because of the increased power losses on the battery resistance due to the higher $i_{\text {OUT }}(t)$ values and on the diodes due to the discontinuous conduction.

\section{Conclusions}

Sizing $C_{0}$ of the output filter of a WPT in order to obtain a stable output brings the designer to oversize its value, adding useless size and load to the drone. The paper shows that the filter can be reduced to a single capacitor, as the inductance can be provided by the battery itself, reducing the on-board circuitry. The power delivered to the battery and the process efficiency were evaluated as a function of the $C_{0}$ value. As in opportunity charging the aim is to maximize the power delivered to the battery, it has been shown that choosing a value of $C_{0}$ that fixes the resonant frequency of the $L C$ filter near the double of the excitation frequency of the WPT system leads to the maximum power transfer. The drawback is a reduced filtering effect on the battery current and a non-optimal value of the efficiency in the power transfer. Instead, if the goal is to maximize the efficiency, a value of $C_{0}$ that sets the resonant frequency of the $L C$-filter close to the WPT excitation frequency leads to the maximum efficiency in power transfer.

\section{References}

[1] M. Lu, M. Bagheri, A. P. James, T. Phung, "Wireless Charging Techniques for UAVs: A Review, Reconceptualization, and Extension," IEEE Access, vol.6, pp.29865-29884, 2018.

[2] T. Campi, S. Cruciani, M. Feliziani, "Wireless power transfer technology applied to an autonomous electric UAV with a small secondary coil," Energies, vol. 11, no. 2, 2018.

[3] Z. Zhang, H. Pang, A. Georgiadis, and C. Cecati, "Wireless Power Transfer - An Overview," IEEE Trans. Ind. Electron., vol. 66, no. 2, pp. 1044-1058, 2019.

[4] W. X. Zhong and S. Y. R. Hui, "Maximum energy efficiency tracking for wireless power transfer systems," IEEE Trans. Power Electron., vol. 30, no. 7, pp. 4025-4034, 2015.

[5] X. Liu, T. Wang, X. Yang, N. Jin, and H. Tang, "Analysis and design of awireless power transfer system with dual active bridges," Energies, vol. 10, no. 10, pp. 1-20, 2017.

[6] J. M. Amanor-Boadu, M. A. Abouzied, and E. Sanchez-Sinencio, "An Efficient and Fast LiIon Battery Charging System Using Energy Harvesting or Conventional Sources," IEEE Trans. Ind. Electron., vol. 65, no. 9, pp. 7383-7394, 2018.

[7] W. Zhang and C. C. Mi, "Compensation topologies of high-power wireless power transfer systems," IEEE Trans. Veh. Technol., vol. 65, no. 6, pp. 4768-4778, 2016.

[8] D. E. Seborg, D. A. Mellichamp, T. F. Edgar, and F. J. Doyle, "Process Dynamics and Control." John Wiley \& Sons, p. 81, 2010.

[9] T. Campi, F. Dionisi, S. Cruciani, V. De Santis, M. Feliziani, and F. Maradei, "Magnetic field levels in drones equipped with Wireless Power Transfer technology," 2016 AsiaPacific Int. Symp. Electromagn. Compat. APEMC 2016, vol. 01, pp. 544-547, 2016.

$\begin{array}{ccc}\text { [10] "Gamry } & \text { Reference } & 3000 . " \\ \text { https://www.gamry.com/potentiostats/reference-3000/. }\end{array}$

[11] "Gamry Echem Analyst." [Online]. Available: https://www.gamry.com/applicationnotes/software-scripting/.

[12] S. De Breucker, K. Engelen, R. D'hulst, J. Driesen "Impact of current ripple on Li-ion battery ageing," World Electric Vehicle Journal, vol. 6, p.0532, 2013. 